\title{
PAPR reduction in OFDM system using combined MCS and DHMT precoding
}

\author{
Mohd Danial Rozaini ${ }^{1}$, Azlina Idris ${ }^{1}$, Darmawaty Mohd Ali ${ }^{1}$, Ezmin Abdullah ${ }^{1}$ \\ ${ }^{1}$ Wireless Communication Technology Group (WiCoT), Faculty of Electrical Engineering, \\ Universiti Teknologi MARA (UiTM), Shah Alam, Selangor, Malaysia
}

\begin{tabular}{|c|c|}
\hline Article Info & ABSTRACT \\
\hline Article history: & \multirow{10}{*}{$\begin{array}{l}\text { Orthogonal Frequency Division Multiplexing (OFDM) has become a } \\
\text { preferable scheme for most high data rate wireless communication standards. } \\
\text { However, the non-linear power amplifier effect experienced in the OFDM } \\
\text { system has increases the peak-to-average power ratio (PAPR). This paper } \\
\text { proposed a Median Codeword Shift (MCS) as a new solution to alleviate the } \\
\text { effect of high PAPR. MCS takes advantage of the codeword structure and bit } \\
\text { position changes through the manipulation of the codeword structure and } \\
\text { permutation process to achieve a low PAPR value. Additionally, the enhanced } \\
\text { version of MCS is also being proposed by merging MCS with the Discrete } \\
\text { Hartley matrix transform (DHMT) precoding method to boost the PAPR } \\
\text { reduction. Simulation results show that MCS is capable of minimizing PAPR } \\
\text { of conventional OFDM with } 24 \% \text { improvement and at the same time } \\
\text { outperform Selective Codeword Shift (SCS) with a } 0.5 \mathrm{~dB} \text { gap. A remarkable } \\
\text { result was also achieved by MCS-DHMT with a } 15.1 \% \text { improvement without } \\
\text { facing any bit error rate (BER) degradation. }\end{array}$} \\
\hline Received Oct 31, 2019 & \\
\hline Revised Dec 15, 2020 & \\
\hline Accepted Dec 23, 2020 & \\
\hline Keywords: & \\
\hline OFDM & \\
\hline PAPR & \\
\hline SCS & \\
\hline MCS & \\
\hline DHMT & \\
\hline
\end{tabular}

Copyright () 2020 Institute of Advanced Engineering and Science. All rights reserved.

\section{Corresponding Author:}

Azlina Idris,

Faculty of Electrical Engineering, Universiti Teknologi MARA (UiTM), 40450, Shah Alam, Selangor, Malaysia.

Email: azlina831@uitm.edu.my

\section{INTRODUCTION}

Orthogonal Frequency Division Multiplexing (OFDM) is a well-known multicarrier modulation technique that has become the fundamental technology for the next generation of wireless communication systems. The popularity of OFDM is influenced by its unique features such as high spectral efficiency, resistance to inter symbol interference (ISI) and robustness against multipath fading. Due to these advantages, OFDM has been adopted in many wireless applications including Wireless Local Area Network (WLAN), Worldwide Interoperability for Microwave Access (WiMAX) and Long Term Evolution (LTE). An OFDM signal comprises multiple numbers of orthogonal narrowband signals transmitted simultaneously at different frequencies. Linear combination of a large number of a narrowband signal having an identical phase will cause the peak value of OFDM signal becomes significantly higher than the average power. Consequently, the OFDM signal is facing a high PAPR value, which is the major limitation of the OFDM system that will contribute to severe nonlinear distortion, efficiency degradation and increasing the cost in practical implementations of the high power amplifier (HPA) [1,2,3]. Therefore, it is crucial to overcome the high PAPR problem in the OFDM system.

The most commonly used techniques to counter high PAPR are categorized into signal distortion, multiple signalling technique and probabilistic, and coding technique [4,5]. Companding, clipping and filtering, peak windowing and peak cancelation are examples of techniques under the signal distortion category. Signal distortion gets less attention from the researcher due to out of band radiations caused by this technique which lead to BER degradation. By far, multiple signalling technique and probabilistic such as selected mapping 
(SLM) [6], partial transmit sequence (PTS) [7], data position permutation (DPP) [8] and selective codeword shift (SCS) [9-12] are the most dominant techniques in PAPR reduction. The aforementioned techniques do not experience BER performance degradation but instead the system complexity increases resulting from the repetitive calculation of IFFTs. As the number of iterations and subcarriers increases, the system complexity will also be increased exponentially. Then the coding technique is applied to code the input data in such a way so that the output of the IFFT blocks will have lower peaks. Block coding, convolutional coding and concatenate coding are some examples of the technique. Unfortunately, the coding technique is less effective in terms of PAPR reduction as it manages to slightly reduce the PAPR value. On the bright side, the BER performance can be improved using error-correcting capabilities.

Selective codeword shift (SCS) is one of the methods that take advantage of bit positioning of a codeward to reduce the PAPR through the shifting process without altering the codeword structure itself. The shifting process enables SCS to generate an alternative codeword in which the lowest PAPR among the alternative codeword will be selected for transmission. This method is better in terms of PAPR reduction and computational complexity compared to SLM and DPP. The computational complexity increases linearly upon increasing the number of IFFT blocks used. On top of that, to the best of the author's knowledge, there is no research is being done to reduce the computational complexity of SCS without compromising its PAPR performance.

Every PAPR reduction method has its limit on how much it can reduce the peak power in the OFDM system. Therefore, to further improve its reduction capability a hybrid method has been proposed as a solution for this issue $[13,14,15]$. Through hybridization, two or more methods can be combined to produce a new method with better PAPR reduction and at the same time will slightly increase the complexity. Precoding is one of the methods that is frequently used in hybridization because of its simplicity to implement. The fundamental of the precoding technique is to multiply each block of modulated symbols with a precoding matrix before the IFFT operation being conducted. The advantage of using a predefined precoding matrix is that the transmission of side information becomes unnecessary to the receiver. But unfortunately, the computational complexity needs to be sacrificed due to the additional multiplication process. Some of the predefined matrices that have been used in precoding based OFDM signals including Walsh-Hadamard matrix transform (WHMT), discrete cosine matrix transform (DCMT) [16], discrete Hartley matrix transform (DHMT) [17] and Vandermonde-like matrix transform (VLMT) [18].

In this paper, a new method is proposed to counteract the high PAPR problem in the OFDM system namely Median Codeword Shift (MCS). MCS is based on the SCS scheme and requires the codeword structure to be altered before the shifting process can be performed. MCS achieves a significant reduction in PAPR without degrading the bit error performance (BER) of the system compared to SCS. To push the limit of MCS reduction capability, the MCS-DHMT method is proposed which is the combination of MCS and DHMT. MCS-DHMT outperforms MCS performance without experiencing any BER degradation.

\section{OFDM SYSTEM MODEL AND PAPR}

In conventional OFDM system, the conversion of information data to OFDM signal begins when the input of binary sequences is grouped into blocks of symbols through the serial-to-parallel converter. The incoming information symbols passed through the digital modulator which converted it into a complex vector of size $N$. The final stage of this whole conversion process ended when Inverse Fast Fourier Transform (IFFT) transform the modulated symbols into an OFDM signal. Figure 1 summarizes the OFDM system.

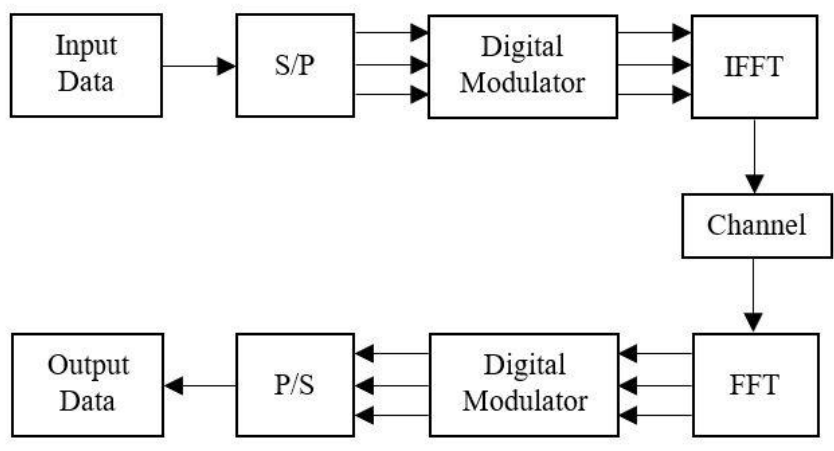

Figure 1. Conventional OFDM transceiver

Mathematically, a complex baseband OFDM signal having $N$ number of subcarriers can be expressed as [9] 


$$
h(t)=\frac{1}{\sqrt{N}} \sum_{k=0}^{N-1} H_{k} \cdot e^{j 2 \pi f_{k} t}
$$

where $N$ represent the number of subcarrier and $T$ is the symbol duration. PAPR is defined as the ratio between maximum peak power to the average power of the complex signal in time domain and can be written as [9]

$$
\operatorname{PAPR}(d B)=10 \log \left(\frac{P_{\text {peak }}}{P_{\text {avg }}}\right)
$$

Therefore, by substituting (1) into (2), the PAPR of the OFDM signal can be rewritten as

$$
\operatorname{PAPR}(d B)=10 \log \left(\frac{\max |h(t)|^{2}}{E|h(t)|^{2}}\right)
$$

where the average power is denoted as $E|h(t)|$. Complementary cumulative distribution function (CCDF) is a method used to evaluate the PAPR reduction capability and it gives information on the probability of the transmitted OFDM symbols exceeds a preset threshold value. The CCDF of the PAPR is given by [15]

$$
C C D F\left(P A P R_{0}\right)=P_{r}\left(P A P R>P A P R_{0}\right)
$$

\section{DHMT PRECODING}

DHMT is a linear transform in which each element of the precoder matrix $M$ having $p$ th row and $q$ th column is defined as follow [17]

$$
M_{p q}=\frac{1}{\sqrt{N}}\left[\cos \left(\frac{2 \pi}{N} p q\right)+\sin \left(\frac{2 \pi}{N} p q\right)\right]
$$

The DHMT matrix engaged with real arithmetic only and has an identical inverse. To be more specific, the DHMT and inverse DHMT matrices are the same $\left(M=M^{-1}\right)$. For that reason, the DHMT precoding method necessitates low implementation complexity. A precoder matrix $M$ having a dimension of $N \times N$ is implemented between digital modulator and IFFT in order to minimize the PAPR. Let $L$ be the baseband modulated symbol and after the multiplication with precoder matrix $M$, a new sequences $\hat{L}$ are produced which can be represented as

$$
\hat{L}=L \times M
$$

Afterward, the signal $\widehat{L}$ will go through $N$-point IFFT operation and this may be expressed as

$$
\hat{L}^{\prime}=\operatorname{IFFT}(\hat{L})=\operatorname{IFFT}(L \times M)
$$

The demodulation at the receiver is a very straightforward process, where the FFT data is multiplied by the inverse DHMT matrix.

When DHMT is applied to the OFDM system, the autocorrelation of the modulated data is lower. Research conducted in [19] concludes that the PAPR of the OFDM signal depends on the aperiodic autocorrelation function (ACF) of the input vector $E$. Suppose that $\varphi(i)$ is the ACF of signal $E$, and thus [18]

$$
\varphi(i)=\sum_{x=0}^{N-1-i} E_{x+i} E_{x}^{*} \quad, \quad i=0,1, \ldots, N-1
$$

where * signify the complex conjugate. Therefore, the PAPR of the transformed signal is bounded [18] 


$$
P A P R \leq 1+\frac{2}{N} \times \gamma
$$

where $\gamma$ is specified as [18]

$$
\gamma=\sum_{i=1}^{N-1}|\varphi(i)|
$$

Where $|\varphi(i)|$ is the absolute aperiodic ACF.

\section{MCS AND MCS-DHMT}

As explained in the previous section, data symbol, $H_{k}$ and orthogonal sinusoids IFFT, $e^{j 2 \pi f_{k} t}$ are the two components involved in the formation of the OFDM signal and to measure PAPR. Data symbol sequences are the complex form of data modulated by the $M$-ary digital modulation QAM to the codeword bits. MCS is a method that manipulates the codeword structure and codeword bits through structure alteration and circulant shift respectively. The purpose of these two manipulations is to allow an alternative codeword to be generated with a low PAPR value. Since MCS is dealing with a codeword, therefore its whole operation will be executed between serial-to-parallel and digital modulator as illustrated in Figure 2.

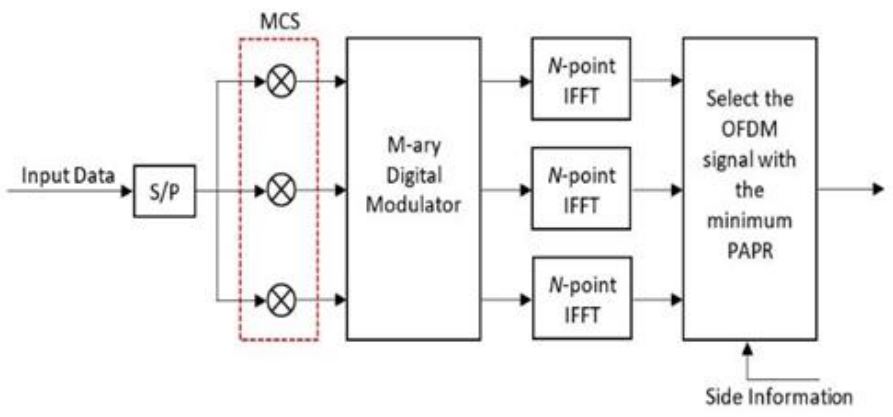

Figure 2. Block diagram of the MCS technique

Referring to Figure 3, the input data $G$ having a total number of bits, $d$ is represented as $G=$ $\left[g_{1}, g_{2}, g_{3}, \ldots, g_{d}\right]$ are divided into sub-blocks, $G_{b}=\left[G_{1}, G_{2}, G_{3}, \ldots, G_{s}\right]$ by serial-to-parallel block where $s=$ $d / e$ is the total number of sub-blocks and $e$ is the number of bits per symbols. The divided codeword $G_{b}$ in $s$ number of sub-block is represented as

$$
G_{b}=\prod_{k=1}^{s} G_{k} \quad, \quad 1 \leq k \leq s
$$

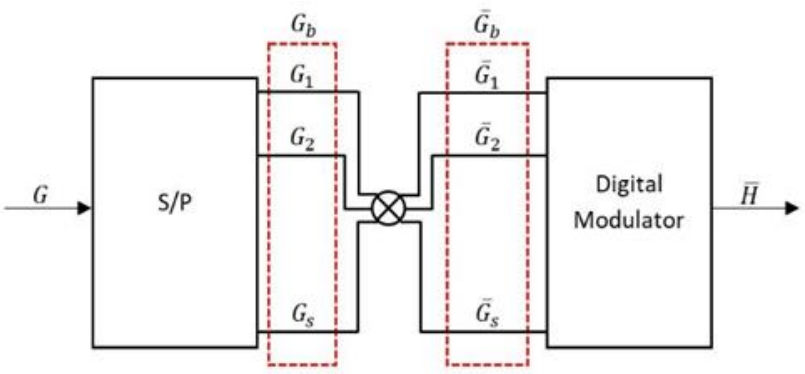

Figure 3. MCS sub-block

where $G_{1}=\left[g_{1}, g_{2}, \ldots, g_{e}\right], G_{2}=\left[g_{e+1}, g_{e+2}, \ldots, g_{2 e}\right]$ and so on until $G_{s}$. The first stage of the MCS method is to modify the codeword structure by splitting it into half and each section is denoted as part A and part B as shown in Figure 4. Next, the altered codeword structure will undergo a shifting process to generate an alternative codeword. The shifting process is performed to both parts of the codeword structure but at different times of execution. For instance, when the shifting process is conducted in part A, part B will stay in its original state until the process is completed and the same goes for part A when part B is performing the shifting process. The total number of shifting, $\sigma$ for both part A and B can be expressed as 


$$
\sigma=(e / 2)-1
$$

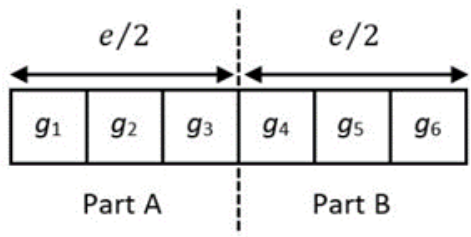

Figure 4. MCS codeword structure

Table 1 shows the bits position after the implementation of the shifting process to both part A and part B for one sub-block. Codeword $G_{1,0}$ represent the original codeword and its initial bits position. The first two alternative codewords generated from the codeword $G_{1,0}$ is produced when the shifting process occurred in part A and codeword $G_{1,1}$ and $G_{1,2}$ symbolized its new bit position. When codeword $G_{1,0}$ go through shifting process in part B, the last two alternative codewords are generated and its bit position is denoted by the codeword $G_{1,3}$ and $G_{1,4}$.

Table 1. Bit arrangement of codeword for MCS technique

\begin{tabular}{cc}
\hline Sub-block codeword bits & Position of bits \\
\hline Codeword $G_{1,0}$ & $g_{1}, g_{2}, g_{3}, g_{4}, g_{5}, g_{6}$ \\
Codeword shift 1, $G_{1,1}$ & $g_{3}, g_{1}, g_{2}, g_{4}, g_{5}, g_{6}$ \\
Codeword shift 2, $G_{1,2}$ & $g_{2}, g_{3}, g_{1}, g_{4}, g_{5}, g_{6}$ \\
Codeword shift 3, $G_{1,3}$ & $g_{1}, g_{2}, g_{3}, g_{6}, g_{4}, g_{5}$ \\
Codeword shift 4, $G_{1,4}$ & $g_{1}, g_{2}, g_{3}, g_{5}, g_{6}, g_{4}$
\end{tabular}

Hence, the new expression for the alternative codeword can be written as $\bar{G}_{b}=\left[\bar{G}_{1}, \bar{G}_{2}, \bar{G}_{3}, \ldots, \bar{G}_{s}\right]$ and the alternative OFDM symbol sequence is given by

$$
\bar{H}=\prod_{i=1}^{s} \bar{G}_{i} \cdot \varpi(t), \quad 1 \leq i \leq s
$$

where $\varpi(t)$ is $M$-ary digital modulation. Thus, the $N$ number of transmitted alternative OFDM signals in the time domain can be represented directly as

$$
\bar{h}(t)=\frac{1}{\sqrt{N}} \sum_{k=0}^{N-1} \bar{H}_{k} \cdot e^{j 2 \pi f_{k} t}
$$

Lastly, the minimum PAPR among the $N$ number of alternative OFDM signals will be selected for transmission. For the data recovery purpose at the receiver, the transmission of side information is necessary alongside the selected alternative OFDM signal. The combination of MCS and DHMT is a viable solution to enhance the MCS PAPR reduction where the multiplication of the precoding matrix is applied between digital modulator and IFFT as shown in Figure 5.

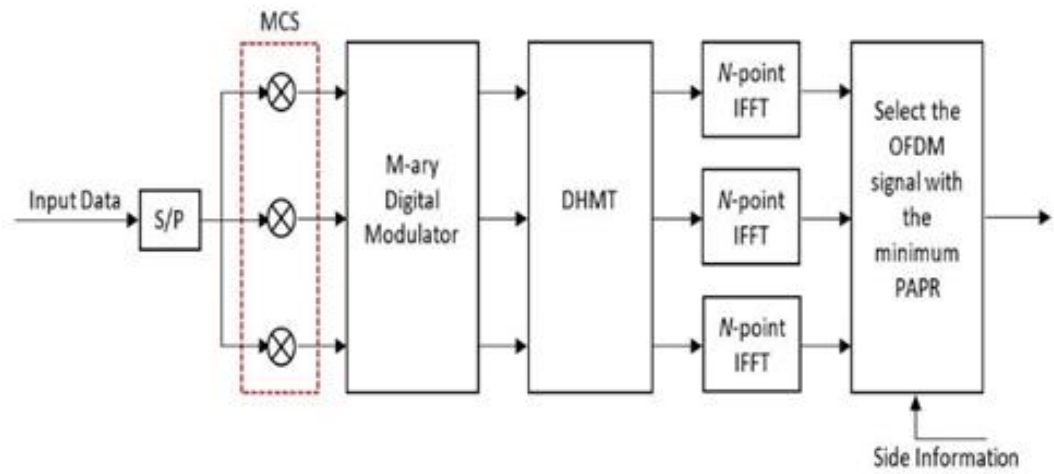

Figure 5. Block diagram of MCS-DHMT technique 
The alternative OFDM symbol sequence $\bar{H}$ in (10) will go through the multiplication process with precoding matrix $M$ that will cause the generation of coded alternative OFDM symbol sequences. The new coded alternative OFDM symbol can be written as

$$
\overline{\bar{H}}=\prod_{x=1}^{N} \bar{H}_{x} \cdot M
$$

Finally, the coded alternative OFDM signals can be represented as

$$
\overline{\bar{h}}(t)=\frac{1}{\sqrt{N}} \sum_{k=0}^{N-1} \overline{\bar{H}}_{k} \cdot e^{j 2 \pi f_{k} t}
$$

\section{RESULTS AND DISCUSSION}

Throughout this section, the performance of MCS and MCS-DHMT will be evaluated in terms of PAPR reduction and BER degradation by using MATLAB. The simulation parameters are based on the 3rd Generation Partnership Project Long Term Evolution (3GPP-LTE) standards [20]. Table 2 outlines the parameters used during the simulation process. To plot the CCDF graph, $10^{4}$ random OFDM symbols are taken into consideration. The number of phase sequences used in SLM was set to be the same as the number of bits per symbol, $e$ used in the proposed technique, to make the computational complexity equal.

Table 2. Simulation parameters

\begin{tabular}{cc}
\hline Parameter & Value \\
\hline Bandwidth (BW) & $1.25 \mathrm{MHz}$ \\
Sampling frequency & $1.92 \mathrm{MHz}$ \\
Sampling time & $5.208 \times 10^{-7} \mathrm{sec}$ \\
IFFT size & 128 \\
Used subcarrier & 76 \\
Modulation technique & $64-\mathrm{QAM}$ \\
Cyclic prefix length & $1 / 4$ \\
Channel model & Rayleigh fading \\
\hline
\end{tabular}

\subsection{PAPR and BER Performance for MCS}

Figure 6 shows the PAPR performance for MCS. The simulation results reveal that MCS has successfully reduced the PAPR value of the OFDM system by $2.6 \mathrm{~dB}$ which is equivalent to $24 \%$ improvement while SLM with 6 different phase sequence and SCS manages to reduce the PAPR value by $1.1 \mathrm{~dB}(10.5 \%$ improvement) and $2.1 \mathrm{~dB}$ (19.2\% improvement) respectively. This result can be explained by the fact that altered codeword structure has improved significantly the PAPR value. Divided codeword structure in MCS is found to cause a reduction in codeword distance hence, it would lead to better PAPR performance. This finding is consistent with [21] which stated that lower codeword distance can improve the reduction of PAPR. Furthermore, MCS also took the advantage of shifting process as a way to improve PAPR performance. The shifting process enables MCS to generate multiple alternative codewords and allows the system to choose the signal with lower PAPR to be transmitted. Since conventional OFDM has only one output signal, therefore the system has no other choice for signal transmission.

Figure 7 compares the BER performance for conventional OFDM, SLM, SCS and MCS. The result, as shown in Table 2, shows that MCS can maintain the same BER performance as conventional OFDM and SCS without any degradation while SLM is found to be the worst one. These findings suggest that MCS has a better resistance against fading channel.

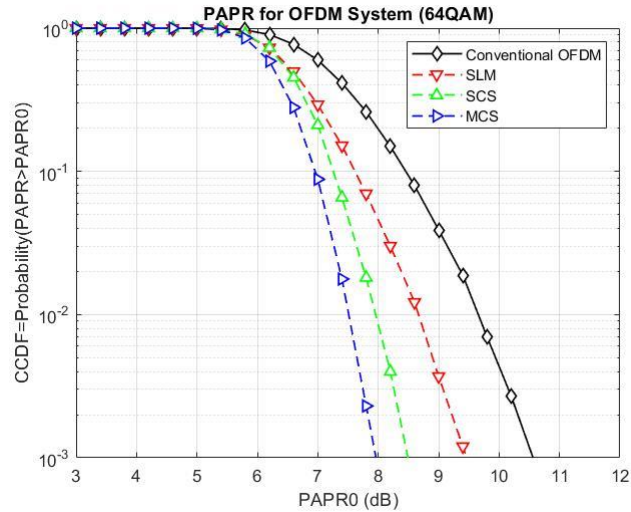

Figure 6. PAPR performance for MCS

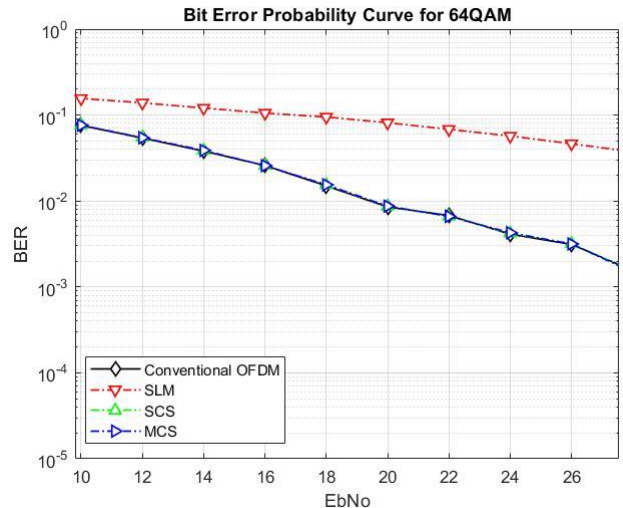

Figure 7. BER performance for MCS 



\subsection{PAPR and BER Performance for MCS-DHMT}

Result shown in Figure 8 prove that DHMT is capable to enhance the PAPR performance of MCS. Comparative analysis demonstrates that as an individual PAPR reduction method, DHMT exceed MCS performance by reducing the PAPR of conventional OFDM by $32.08 \%$ while MCS manage to achieved $25.47 \%$ of reduction only. The $0.7 \mathrm{~dB}$ differences in PAPR reduction performance between DHMT and MCS is because of DHMT reduces the autocorrelation of the modulated data as a way to achieve better PAPR performance. It is clear from (9) and (10) that the input vector that possess lower $\gamma$ will lead to better PAPR reduction. Meanwhile, MCS relies on codeword manipulation in order to obtain an OFDM signal that has a satisfactory PAPR value. MCS-DHMT proven to be a compatible match between MCS and DHMT whereby its PAPR performance is almost twice as compared to the MCS itself with $40.57 \%$ improvement. This outstanding performance of MCS-DHMT is driven by the capability of DHMT to lower the autocorrelation of alternative modulated OFDM symbols. Meanwhile, the PAPR performance of SLM and SCS is still far behind MCSDHMT with a reduction of only $1.1 \mathrm{~dB}$ and $2.1 \mathrm{~dB}$ respectively.

Figure 9 presents the BER performance for the conventional OFDM, SLM, SCS, MCS, DHMT and MCS-DHMT in Rayleigh fading channel. The simulation result shows that DHMT is comparable to SCS and MCS by not experience BER degradation but unfortunately, not for SLM which has worse BER performance. As mentioned in [22], all precoding matrix including DHMT are unitary, in which they satisfy Parseval's theorem that state energy is conserved when a signal is converted from the frequency domain to the time domain. Due to this property, DHMT is able to prevent loss of total energy and this condition cause no degradation in the BER performance. As expected, this property also applicable to MCS-DHMT which lead to identical BER performance as MCS and DHMT.

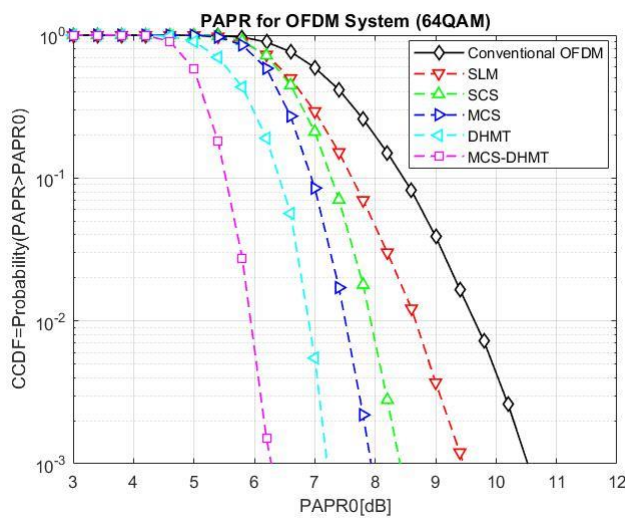

Figure 8. PAPR performance for MCS-DHMT

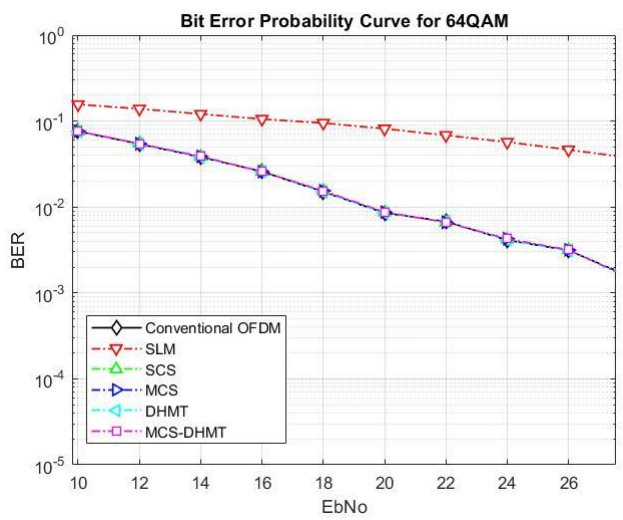

Figure 9. BER performance for MCS-DHMT

\section{CONCLUSION}

In this paper, a new method under multiple signalling technique and probabilistic scheme called MCS is proposed and thoroughly investigated as a countermeasure to reduce the PAPR in OFDM transmission. Computer simulations demonstrate that MCS successfully fulfill its objective and managed to reduce the PAPR of conventional OFDM by $24 \%$ without having any BER degradation. On top of that, the effectiveness of MCS in minimizing PAPR also surpasses SCS with $4.8 \%$ improvement. Although DHMT has slightly increases the computational complexity of the system due to additional stage of multiplication, but it is still worth it, when the simulation result of MCS-DHMT indicates that $15.1 \%$ of improvement can be achieved through the combination of MCS and DHMT. This achievement is considered an acceptable trade-off between computational complexity and PAPR reduction.

\section{ACKNOWLEDGMENTS}

We are grateful to the Faculty of Electrical Engineering, Universiti Teknologi MARA (UiTM) Shah Alam, Selangor for providing the insight and expertise that greatly assist this research.

\section{REFERENCES}

[1] H. S. Hee and L. J. Hong, "An Overview of Peak-to-Average Power Ratio Reduction Techniques for Multicarrier Transmission,” IEEE Wireless Communications, vol. 12, no. 2, pp. 56-65, 2005. 
[2] A. Kumar and M. Gupta, "A Review on OFDM and PAPR Reduction Techniques," American Journal of Engineering and Applied Sciences, vol. 8, no. 2, pp. 202-209, 2015.

[3] T. Jiang and Y. Wu., "An Overview: Peak-to-Average Power Ratio Reduction Techniques for OFDM Signals," IEEE Transactions on Broadcasting, vol. 54, no. 2, pp. 257-268, 2008.

[4] F. Sandoval, G. Poitau, and F. Gagnon, "Hybrid Peak-to-Average Power Ratio Reduction Techniques: Review and Performance Comparison," IEEE Access, vol. 5, pp. 27145 - 27161, 2017.

[5] K. Anoh, B. Adebisi, K. M. Rabie, and C. Tanriover, "Root-Based Nonlinear Companding Technique for Reducing PAPR of Precoded OFDM Signals," IEEE Access, vol. 6, pp. 4618-4629, 2017.

[6] S.-Y. Zhang and B. Shahrrava, "A Selected Mapping Technique Using Interleavers for PAPR Reduction in OFDM Systems," Wireless Personal Communications, vol. 99, no. 1, pp. 329-338, 2018.

[7] K. Chung, H. Chen, and T. Yang, "Low Complexity PTS Algorithms with Error Correction Capability in OFDM Systems," 2015 Seventh International Conference on Ubiquitous and Future Networks, pp. 254-256, 2015.

[8] J. Wen, S. Lee, and C. Kung, "SLM Based Data Position Permutation Method for PAPR Reduction in OFDM Systems," Wireless Communications and Mobile Computing, vol. 13, no. 11, pp. 985-997, 2013.

[9] E. Abdullah, A. Idris, and A. Saparon, "Minimizing High PAPR in OFDM System using Circulant Shift Codeword," Jurnal Teknologi, vol. 78, no. 2, pp. 135-140, 2016.

[10] E. Abdullah and N. M. Hidayat, "SCS-SLM PAPR Reduction Technique in STBC MIMO-OFDM Systems," 2017 7th IEEE International Conference on Control System, Computing and Engineering (ICCSCE), pp. 104-109, 2017.

[11] E. Abdullah, A. Idris, and A. Saparon, "Modified Selective Mapping Scheme with Low Complexity for Minimizing High Peak-Average Power Ratio in Orthogonal Frequency Division Multiplexing System," AIP Conference Proceedings, pp. 1-7, 2016.

[12] E. Abdullah, A. Idris, and A. Saparon, "Performance Evaluation of Modified SLM Technique in OFDM System using Selected Codeword Shift," MATEC Web of Conferences, vol. 75, 2016.

[13] D. D. Prasad and M. V. N. Rao, "A Hybrid Companding Transform Technique for PAPR Reduction of OFDM Signals," 2015 13th International Conference on Electromagnetic Interference and Compatibility (INCEMIC), pp. 263-268, 2015.

[14] X. He, H. Yan, J. He, M. Cai, and Z. Jing, "A Combined PTS-Companding Scheme for PAPR Reduction in OFDM System," 2017 IEEE 2nd Advanced Information Technology, Electronic and Automation Control Conference (IAEAC), pp. 196-199, 2017.

[15] Z. T. Ibraheem, M. M. Rahman, Y. Fazea, and K. K. Ahmed, "PAPR Reduction in OFDM Signal by Incorporating Mu-Law Companding Approach into Enhanced PTS Scheme," Journal of Optical Communications, 2018.

[16] I. Baig, M. Ayaz, and V. Jeoti, "On the Peak-to-Average Power Ratio Reduction in Mobile WiMAX: A Discrete Cosine Transform Matrix Precoding Based Random-Interleaved Orthogonal Frequency Division Multiple Access Uplink System," Journal of Network and Computer Applications, vol. 36, no. 1, pp. 466-475, 2013.

[17] A. Ali Sharifi, "Discrete Hartley Matrix Transform Precoding-Based OFDM System to Reduce the High PAPR," ICT Express, vol. 5, no. 2, pp. 100-103, 2019.

[18] M. M. Hasan, "VLM Precoded SLM Technique for PAPR Reduction in OFDM Systems," Wireless Personal Communications, vol. 73, no. 3, pp. 791-801, 2013.

[19] C. Tellambura, "Upper Bound on Peak Factor of N-Multiple Carriers," Electronics Letters, vol. 33, no. 19, pp. 1608, 1997.

[20] T. D. Chiueh and P. Y. Tsai, OFDM Baseband Receiver Design for Wireless Communications. 1st ed. Singapore: John Wiley and Sons (Asia) Pte Ltd, 2007.

[21] K. G. Paterson and V. Tarokh, "On the Existence and Construction of Good Codes with Low Peak-to-Average Power Ratios," IEEE Transactions on Information Theory, vol. 46, no. 6, pp. 1974-1987, 2000.

[22] C.-Y. Hsu and H.-C. Liao, "Generalised Precoding Method for PAPR Reduction with Low Complexity in OFDM Systems," IET Communications, vol. 12, no. 7, pp. 796-808, 2018. 